\title{
EL USO DE אהב EN DEUTERONOMIO 10:12-22 COMO REVELACIÓN DEL CARÁCTER DE DIOS
}

\author{
Emmer Chacón
}

\section{Introducción}

La erudición crítica $^{1}$ ve el vocabulario de amor en Deuteronomio como una formalidad en el contexto de un pacto del tipo suzerano-vasallo. Esta perspectiva se basa en estudios comparativos entre el pacto de Deuteronomio y la literatura del Antiguo Cercano Oriente. Desde esta pers-

${ }^{1}$ Véase P. J. J. S. Els, "אָָָָ", New International Dictionary of the Old Testament Theology and Exegesis (Grand Rapids, MI: Zondervan, 1997), 3:283-90. Els se enfoca en aquellos pasajes donde \$ָָ tiene a Dios como objeto directo en Deuteronomio, así como en la literatura deuteronomista, y concluye que Deuteronomio tiene una dependencia literaria de esas fuentes. Este artículo no está de acuerdo con el enfoque de criticismo de la redacción seguido por Els. La presencia de temas comunes en Deuteronomio y en otros textos del AT, así como también la presencia de intertextualidad, se puede entender de manera inversa; es decir, que estos otros textos citan y/o aluden al Deuteronomio como fuente a partir de la cual desarrollan su teología. Véase Ronald Bergey, "The Song of Moses (Deuteronomy 32.1-43) and Isaianic Prophecies: A Case of Early Intertextuality?", Journal for the Study of the Old Testament 28, no. 1 (2003): 53. Luego de su análisis Bergey concluye que Dt 32:1-43 fue la fuente de los materiales deuteronómicos de Isaías y no al revés. Véase también William L. Moran, "The Ancient Near Eastern Background of the Love of God in Deuteronomy”, The Catholic Biblical Quarterly" 25 (1963): 77-87. Brian Britt analiza el amor pactual desde la perspectiva de חסד en Brian Britt, "Unexpected Attachments: A Literary Approach to the Term חסד in the Hebrew Bible", Journal for the Study of the Old Testament 27, no. 3 (2003): 289-307. Para una perspectiva moral véase John H. Walton, "Deuteronomy: An Exposition of the Spirit of the Law Grace", Theological Journal 8, no. 2 (1987): 213-25. 
pectiva, ¿qué es lo que tiene para decir el contexto interno en Dt 10:12-22 acerca del amor de Dios hacia su pueblo y del pueblo hacia Dios?

Deuteronomio es el libro del Pentateuco que más usa la raíz verbal אָד.. Esta raíz verbal se usa 22 veces en Deuteronomio ${ }^{3}$ en contraste con 14 apariciones en Génesis, 2 en Éxodo, 2 en Levítico y ninguna en Números. La elección de este pasaje se basa en el hecho de que en este texto aparece 4 veces la raíz אָז siendo este pasaje el que posee la concentración más elevada de apariciones. Otros tienen 3 como máximo, como los capítulos 11 y 30. Además, Dt 10:12-22 se encuentra en el contexto de la conmemoración de la ocasión en la cual Moisés reescribió la ley.

Este trabajo utilizará un procedimiento exegético a fin de evaluar la función de la raíz verbal אָהב en Dt 10:12-22. Además, prestará atención al uso de esta raíz en el resto del libro a fin de ver la función de este lexema en Dt 10:12-22 a la luz de su contexto interno más amplio.

En primer lugar, es necesario establecer los límites literarios del pasaje y evaluar su situación textual. Segundo, como el texto es parte del segundo discurso de Deuteronomio (4:44-26:19), se necesita analizar su estructura retórica interna a fin de evaluar la función de la raíz אָָָ en el pasaje. Finalmente, se analiza la contribución teológica del texto.

\section{Delimitación de Deuteronomio 10:12-22}

Deuteronomio 10:12-22 es parte del segundo y más

${ }^{2}$ Francis Brown, S. R. Driver y Charles A. Briggs, A Hebrew and English Lexicon of the Old Testament With an Appendix Containing the Biblical Aramaic, based on the lexicon of William Gesenius (1907), s.v. "אָָ.".

${ }^{3}$ Estas referencias son: Dt 4:37; 5:10; 6:5; 7:9, 13; 10:12, 15, 18, 19; 11:1, 13, 22; 13:4; 15:16; 19:9; 21:15 (2x); 23:6; 30:6, 16, 20. 
extenso discurso del libro del que forma parte: 4:44-26:19. ${ }^{4}$ A fin de determinar los límites de Dt 10:12-22 es importante decir que en Dt 12:1 comienza una clase de inclusio literario-temático con una sección cúltica que marca un cambio en el tema que se ha tratado hasta Dt 11:32. ${ }^{5}$ Además, la ex-

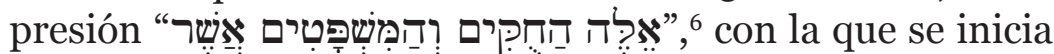
12:1, 7 señala una transición mayor en el discurso. ${ }^{8} \mathrm{Al}$ final de Dt 10:11 hay una petuhah ( $\Xi$ ) que señala el inicio de una nueva sección. Dentro de Dt 10:12-11:32 se encuentra un ziah al final de 10:22 y la división de capítulo. Al final de 11:7

${ }^{4}$ Desde esta perspectiva la estructura del libro es la siguiente: prólogo, 1:1-5; primer discurso, 1:6-4:43; segundo discurso, 4:44-26:19; tercer discurso, 27:1-28:68; cuarto discurso, 29:1-30:20; epílogo, 31:1-34:12. Véase Gerhard Pfandl, "The Soteriological Implications of the Cities of Refuge," en Inicios, paradigmas y fundamentos: Estudios teológicos y exegéticos en el Pentateuco, Serie monográfica de Estudios Bíblicos y Teológicos de la Universidad Adventista del Plata 1, ed. Gerald Klingbeil (Libertador San Martín, Entre Ríos, Argentina: Editorial Universidad Adventista del Plata, 2004), 236n35.

${ }^{5} \mathrm{El}$ otro pasaje cúltico se encuentra en Dt 26, por lo tanto Dt 12-26 es una unidad literaria dentro de Dt 4:44-26:19. Construcciones similares se encuentran presentes en Éx 20:22-23:19, que comienza y termina enmarcado en lenguaje cúltico (20:22-26; 23:10-19), así como también Lv 17-26. Esto muestra el énfasis más bien religioso antes que secular de estos pasajes en diametral diferencia con los documentos cuneiformes. Además, a lo largo del Pentateuco se encuentran evidencias de unidad literaria cuando se presentan estructuras literarias similares en diversas secciones. Véase Joe M. Sprinkle, "The Book of the Covenant": A Literary Approach, Journal for the Study of the Old Testament Supplemental Series 174 (Sheffield: JSOT Press, 1994), 37.

${ }^{6}$ Los textos de la Escritura Hebrea son de K. Elliger y W. Rudolph, eds., Biblia Hebraica Stuttgartensia, 5ta ed. rev. (Stuttgart: Deutsche Bibelgesellschaft, 1997).

7La forma indefinida de אליל se usa en Deuteronomio solamente en 1:1, 4:5, 12:1, 27:12 y 28:69.

${ }^{8}$ Véase Jason Shane DeRouchie, "A Call to Covenant Love: Text Grammar and Literary Structure in Deuteronomy 5-11" (PhD. diss., Southern Baptist Theological Seminary, 2005), 53. 
hay otra ziah. Se encuentran setumah - - al final de 11:9, 11:12, 11:21; 11:25 and 11:28. Por lo tanto, el análisis para delimitar el texto y el movimiento temático muestran los límites de Dt 10:12-22 en el contexto más extenso del discurso en el que se encuentra. Además el pasaje se encuentra enmarcado entre los vv. 12a y 22b que inician con la expre-

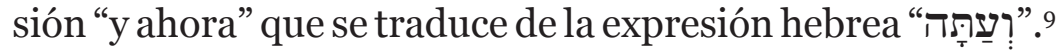
De acuerdo a J. Gordon McConville, esta expresión en 10:12a señala - de forma similar a su uso en Dt 4:1, el otro

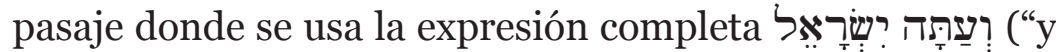
ahora Israel") - el movimiento de "la reminiscencia histórica hacia el mandato. En ambos casos la reminiscencia histórica se ha focalizado en la gracia de Dios en la vida de Israel a pesar de la dificultad experimentada por este último en guardar el pacto". ${ }^{10}$ Deuteronomio 10:12-22 es visto como una unidad temática que llama "a amar y servir a Dios, desarrollando así [Dt] 6:5". ${ }^{11}$ McConville detecta cinco elementos que en Dt 10:12-22 miran hacia atrás, hacia la historia previa del pueblo de Dios, hacia su futuro próximo, en la medida que se aproximan a la tierra prometida, y hacia su futuro lejano en la medida en que el contenido del pasaje se refleja en el anuncio del nuevo pacto en Dt 30 y en la tradición profética como en Mi 6:8. ${ }^{12}$ El Pentateuco como un todo comparte esta naturaleza profética y escatológica que John H. Sailhamer ha detectado y otros han elabora-

${ }^{9}$ Usado en Dt 4:1; 5:25; 10:12, 22; 26:10; 31:19.

${ }^{10}$ J. Gordon McConville, Deuteronomy, Apollos Old Testament Commentary 5 (Leicester: Apollos, 2002), 198-9. Véase John H. Sailhamer, The Pentateuch as Narrative (Grand Rapids, MI: Zondervan, 1992), 442-4.

${ }^{11}$ McConville, Deuteronomy, 198. Véase Sailhamer, The Pentateuch as Narrative, 444.

${ }^{12}$ McConville, Deuteronomy, 197-8. 
do. ${ }^{13}$ El Pentateuco lee la historia del pueblo de Dios desde una perspectiva profética, tipológica y escatológica. Al hacerlo, el Pentateuco establece el programa de Dios para su pueblo en el futuro cercano y lejano a la vez que es perfectamente consciente de la historia del pueblo tanto en el pasado como en relación con el futuro cercano y lejano.

Estas breves consideraciones previas establecen el contexto literario inmediato de nuestro pasaje y proveen las primeras conexiones intertextuales y teológicas. Con estas consideraciones como trasfondo, es tiempo de proceder con el análisis del texto mismo.

\section{אָָהָ en Deuteronomio 10:12-22}

El texto de Dt 10:12-22 se puede subdividir internamente en tres o cuatro subsecciones de acuerdo a la forma en la que se evalúa el flujo de su temática interna. En los vv. 12-13 y 2022 está presente la orden de temer al Señor con su respectiva justificación. Los vv. 14-19 constituyen una descripción del Señor. McConville y Duane L. Christensen coinciden en percibir una división en los vv. 14-16 y 17-19.14 Cuando se analice su contenido, esta subdivisión será visible más claramente.

\section{Deuteronomio 10:12-13}

Este flujo temático muestra una invocación de apertura, presentando a "Israel" en una posición enfática fron-

${ }^{13}$ Sailhamer, The Pentateuch as Narrative, 37 . Véase Richard M. Davidson, "The Eschatological Literary Structure of the Old Testament", en Creation, Life, and Hope: Essays in Honor of Jacques B. Doukhan, ed. Jiří Moskala (Berrien Springs, MI: Old Testament Department, SDA Theological Seminary, Andrews University, 2000), 349-66.

${ }^{14}$ Duane L. Christensen, Deuteronomy 1-11, WBC 6A (Dallas, TX: Word Books, 1998), 201, 205; McConville, Deuteronomy, 198-201. 
tal en 12a ("Y ahora Israel"), donde Dios reúne a su pueblo y entonces le declara su ideal. Una vez que se establece la cuestión, Dios comienza a declarar su programa para su pueblo con cinco construcciones infinitivas verbales. Los requerimientos para el pueblo incluyen: temer (ליריאָד) al

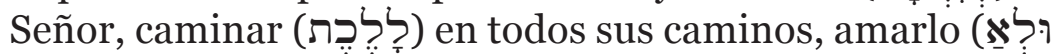

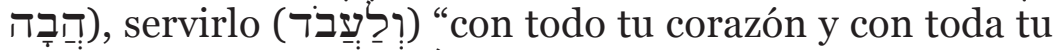

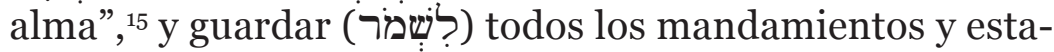
tutos del Señor. ¿Cómo pueden todos estos imperativos llegar a ser una realidad en la experiencia personal del creyente? El resto de la sección lo clarificará.

Esta subsección funciona como un resumen que anticipa el cuadro completo en su forma final en tanto que el resto del pasaje clarifica el cómo del procedimiento para arribar a esa instancia. Joe M. Sprinkle ha identificado en la secuencia de eventos en Éx 19-24 la presencia de una técnica literaria de sinopsis/reanudación/expansión. Esta técnica literaria le permite al escritor bíblico narrar el relato dos veces y, en la segunda ocasión, expandirlo e incluso usar otro punto de vista. Esta técnica literaria se ha detectado en otros pasajes en el AT. ${ }^{16}$ Esta es la primera vez que la raíz wָ ș se usa en este pasaje.

Ahora es conveniente mirar al uso de la raíz en Deuteronomio. De los 22 usos de אזָ en Deuteronomio, 5 tienen al Señor como sujeto (Dt 4:37; 7:13; 10:15, 18; 23:6). De estos, 2 tienen al pueblo de Dios como objeto $(7: 13 ; 23: 6), 1$ tiene al extranjero (10:18) y 2 tienen a los antepasados ("padres", 4:37;

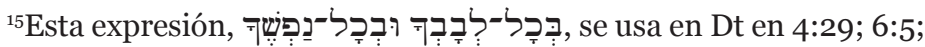
10:12; 26:16; 30:2, 6, 10.

${ }^{16}$ Sprinkle, "The Book of the Covenant", 19. Uno de los pasajes donde se usa esta técnica es Dt 30:1-20, pasaje que tiene conexiones con Dt 10:1222 como se verá más tarde en este artículo. Deuteronomio 30:1-5 despliega el escenario final y completo en tanto que 30:6-14 despliega los detalles y 30:15-20 efectúa la recapitulación, análisis adicional y conclusión. 
10:15). A su vez, 13 tienen al pueblo de Dios como sujeto y de estos 12 tienen a Dios como objeto. ${ }^{17}$ En otros casos el sujeto es más genérico e indefinido como parte de una ley apodíctica, es decir, un esposo (Dt 21:15 [2x]; 21:16) o un esclavo (15:16).

$\mathrm{Al}$ observar a aquellos usos que se encuentran antes de Dt 10, la primera situación es Dt 4:37. En este texto, Dios dice que amó a los padres del pueblo y por esta razón escogió a sus descendientes. A su vez ellos, como pueblo, fueron sacados de Egipto $^{18}$ en cumplimiento de las promesas de Dios a sus padres. Este tema se presenta de vuelta en Dt 10:15. Deuteronomio 5:10 es parte del segundo de los Diez Mandamientos y establece el tenor de la clase de relación que Dios espera de los que se vinculan con Él. Deuteronomio 6:5 es la raíz textual clara del tema desarrollado en Dt 10:12 así como también en varias otras secciones del libro de Deuteronomio e incluso del libro de Josué. ${ }^{19}$ Deuteronomio 7:9 se elabora a la luz de Dt 5:10 y claramente en un contexto con vocabulario pactual. ${ }^{20}$ En Dt 7:13 la raíz אָָָ se usa en el contexto de las promesas pactuales (vv. 13-16). En todos estos pasajes la raíz rị retrata una relación pactual profunda entre el Señor y su pueblo. Además, se puede percibir una relación intertextual estrecha entre estos pasajes.

\section{Deuteronomio 10:14-16}

Esta sección describe quién es el Señor y lo que ha hecho en la naturaleza y en favor de su pueblo de forma tal que el pueblo se sienta impelido a responder circuncidando su

${ }^{17}$ Véase Dt 5:10; 6:5; 7:9; 10:12, 19; 11:1, 13, 22; 13:4; 19:9; 30:6, 16, 20.

${ }^{18}$ La raíz מְְִִרָרי.ם (Egipto) se usa al menos 50 veces en Deuteronomio.

${ }^{19}$ Como en Dt 4:29; 11:1, 13, 22; 13:4; 19:9; 26:16; 30:2, 6, 10, 16, 20; Jos 22:5; 23:11, 14. Véase McConville, Deuteronomy, 142.

20Ibíd., 157-9. 
corazón y no endureciendo más su cerviz. La base sobre la cual Dios elije al pueblo es el amor por sus padres. Por lo tanto, "Israel debería ofrecer a Dios una obediencia futura sobre la base de los pasados actos de gracia sobre ellos". ${ }^{21}$ ¿Cómo puede el pueblo ser capaz de circuncidar su corazón de forma tal que puedan amar al Señor y no endurecer ya más su cerviz? La respuesta se encuentra en el texto paralelo de Dt 30:6. Este texto que presenta el mismo concepto en su contexto, Dt 30:1-20, clarifica el procedimiento y su orden de forma tal que el pueblo sea capaz de amar a Dios, obedecerlo $^{22}$ y aferrarse a Él. El texto muestra que es Dios quien circuncida el corazón del pueblo y este es el hecho que hace posible que el pueblo ame a Dios, lo obedezca y se aferre a Él. Ese es el orden correcto. Por lo tanto, no hay legalismo ni formalismo. Hay un flujo de amor de parte de Dios hacia su pueblo que es previo y una precondición para que el pueblo ame al Señor. La acción de Dios sobre su corazón habla de una transformación que se encuentra más allá de los propios recursos del pueblo. ${ }^{23}$ Sólo Dios quien es el propietario del cielo y la tierra, y todo lo que hay en ellos, el Creador, es capaz de recrear el corazón de su pueblo para que amen, obedezcan y sirvan a su Señor.

\section{Deuteronomio 10:17-19}

Esta subsección muestra una triple descripción del Señor. Primero, quién es el Señor, en el v. 17a ("Porque Jehová vuestro Dios es Dios de dioses, Dios grande, poderoso y te-

${ }^{21}$ Bruce K. Waltke y M. O'Connor, An Introduction to Biblical Hebrew Syntax (Winona Lake, IN: Eisenbrauns, 1990), 532.

${ }^{22}$ Enrique Nardoni, "Normas de justicia en las leyes de la alianza", Revista Bública 58, no. 2 (1996): 82-3.

${ }^{23}$ Rebecca Abts Wright, “The Impossible Commandment”, Anglican Theological Review 83, no. 3 (2001), 579-84. 
mible"). Segundo, qué es lo que Él no hace, 17b ("que no hace acepción de personas, ni toma cohecho”). Tercero, qué es lo que sí hace, v. 18 ("que hace justicia al huérfano y a la viuda; que ama también al extranjero dándole pan y vestido”). A continuación se muestra lo que el pueblo debe hacer como una respuesta al modelo ético de Dios. Debido a que Dios ama al extranjero (v. 18b), el pueblo debe amarlo también (v. 19a) porque ellos fueron también extranjeros en la tierra de Egipto (v. 19b). Dios muestra su misericordia al amar y proveer para el extranjero. El pueblo de Dios, quien fue extranjero en Egipto y ha gozado de la liberación, protección y provisión de Dios, debe responder de la misma forma al extranjero, al huérfano y a la viuda. ${ }^{24}$ Por lo tanto, en esta sección la relación de amor entre Dios y su pueblo se entiende como teniendo una manifestación vertical y otra horizontal. La relación de amor con Dios debe ser visible en la vida diaria y en la ética del pueblo de Dios. La memoria de las maravillas de Dios en favor de su pueblo en la historia de la salvación, debe impactar la experiencia presente y futura.

\section{Deuteronomio 10:20-22}

Esta subsección regresa conceptualmente a Dt 10:1213 pero lo elabora más. Así como el término "Israel" se encuentra en una posición frontal en Dt 10:12a, ahora el Señor está en esa posición en la conclusión de la sección en el v. 20a. Los verbos se encuentran en infinitivo en los vv. 12-13, mostrando la idea de propósito. Pero en esta subsección los verbos están en imperfecto, enfatizando el futuro. Se enfatiza el propósito de Dios para su pueblo. Esta es la clase de vida que Dios desea que su pueblo viva; una vida centrada en Dios y no en ellos. Se retrata un modelo de vida teocén-

${ }^{24}$ Nardoni, "Normas de justicia en las leyes de la alianza”, 107-9. 
trico. ${ }^{25}$ Dios desea que su pueblo viva en respuesta al modelo ético de Dios, en una profunda relación personal con Él. ${ }^{26}$ Esta perspectiva contrasta agudamente con la perspectiva de Susan Ackerman acerca de un concepto de amor en Deuteronomio basado políticamente. ${ }^{27}$

Los eventos del pasado se enfatizan una vez más al traer a la memoria el registro del Éxodo en el v. 22, cuando Dios llama la atención de su pueblo al cumplimiento de la promesa de Dios a Abraham a quien dijo que su descendencia sería tan numerosa "como las estrellas del cielo en multitud" (Gn 15:5). ${ }^{28}$ Dios amó a su pueblo antes de que este existiera o lo amara a Él.

\section{Conclusión}

Esta breve revisión exhibe a Dt 10:12-22 como una muestra de cómo se usa la raíz א̦ en todo el libro de Deuteronomio. El amor del pueblo hacia Dios es el requisito para una vida teocéntrica, que es lo que Dios desea para su pueblo. Este amor del pueblo hacia Dios tiene un antecedente: Dios ama a su pueblo mucho antes de que este existiera. Dios amó a los ancestros del pueblo -Abraham, Isa-

${ }^{25}$ DeRouchie, "A Call to Covenant Love", 263-5.

${ }^{26} \mathrm{En}$ cuanto a esta dimension personal del amor en Deuteronomio, véase Jacqueline E. Lapsley, "Feeling Our Way: Love for God in Deuteronomy", The Catholic Biblical Quarterly 65 (2003): 350-69.

${ }^{27}$ Susan Ackerman, "The Personal Is Political: Covenantal and Affectionate Love ('ĀHĒB, 'AHĂBÂ) In the Hebrew Bible" Vetus Testamentum 52, no. 4 (2002): 457. Para una aproximación al amor de Dios en el AT como personal, véase Hermann Spieckermann, "God's Steadfast Love Towards a New Conception of Old Testament Theology", Biblica 81 (2000): 305-27.

${ }^{28} \mathrm{La}$ expresión se usa en Gn 22:17; 26:4; Éx 32:13; Dt 1:10; 10:22; 28:62; $1 \mathrm{Cr} 27: 23$. 
ac y Jacob- y a causa de ese amor Dios amó al pueblo. Dios tiene un programa para su pueblo pero a fin de cumplir esta agenda tiene que pasar algo primero: Dios circuncidará los corazones de su pueblo; Dios lo transformará. Esta trasformación hará posible que el pueblo tema a Dios, camine en sus caminos, lo ame, le sirva y guarde todos sus mandamientos y estatutos. También hará posible que el pueblo se aferre a Dios. Esta relación vertical entre Dios y su pueblo se verifica en un compromiso recíproco total y personal. Se ve como una respuesta agradecida del pueblo a causa de los actos del Dios todopoderoso en su favor en la historia remota y reciente.

La dimensión vertical de la relación entre Dios y su pueblo debe afectar y reflejarse en la experiencia ética horizontal del pueblo. Dios mismo muestra su propio ejemplo cuando trata con las necesidades del huérfano, la viuda y el extranjero. Este mismo ejemplo debe repetirse en la vida y experiencia diaria de Israel. Los actos poderosos de Dios en la historia de la salvación deben ser un motivo de gratitud para su pueblo que se vuelque hacia el necesitado, el huérfano, la viuda y el extranjero.

Este estudio ha encontrado que los datos sugieren que el uso de la raíz אָ̣ en Dt 10:12-22 retrata una experiencia profunda y personal entre Dios y su pueblo y no sólo una respuesta legal-pactual formal al ofrecimiento unilateral de Aquel que protege al siervo obediente. Los datos sugieren la presencia de un programa de Dios para su pueblo. Esta agenda implica un estilo de vida que se caracteriza por tener a Dios como su centro y que conlleva una trasformación previa del individuo a través de la acción de Dios en él/ella. Esta vida se vive en dimensiones interpersonales recíprocas tanto de forma vertical como horizontal.

Además, la forma en la que la raíz אָָָ funciona en Dt 10:12-22 parece implicar conexiones intertextuales. El 
posible uso de intertextualidad en relación con esta raíz en Deuteronomio e incluso fuera del libro puede ser motivo de otro estudio.@

\author{
Emmer Chacón \\ emmerc@um.edu.mx \\ Universidad de Montemorelos \\ Nuevo León, México \\ Recibido: 10/11/2016 \\ Aceptado: 27/04/17
}

